\title{
Estimating the relative water content of leaves in a cotton canopy
}

\section{Vern Vanderbilt, Craig Daughtry, Meredith Kupinski, Christine Bradley, Andrew French, et al.}

Vern Vanderbilt, Craig Daughtry, Meredith Kupinski, Christine Bradley, Andrew French, Kevin Bronson, Russell Chipman, Robert Dahlgren, "Estimating the relative water content of leaves in a cotton canopy," Proc. SPIE 10407, Polarization Science and Remote Sensing VIII, $104070 Z$ (30 August 2017); doi: 10.1117/12.2274502

SPIE Event: SPIE Optical Engineering + Applications, 2017, San Diego, California, United States 


\title{
Estimating the relative water content of leaves in a cotton canopy
}

\author{
Vern Vanderbilt ${ }^{*}$, Craig Daughtry ${ }^{\mathrm{b}}$, Meridith Kupinski ${ }^{\mathrm{c}}$, Christine Bradley ${ }^{\mathrm{c}}$, Andrew \\ French $^{\mathrm{d}}$, Kevin Bronson $^{\mathrm{d}}$, Russell Chipman ${ }^{\mathrm{c}}$, Robert Dahlgren ${ }^{\mathrm{e}}$ \\ ${ }^{a}$ NASA Ames Research Center, Moffett Field, CA, USA; ${ }^{b}$ USDA-ARS Hydrology \& \\ Remote Sensing Lab, Beltsville, MD, USA; ${ }^{\circ}$ College of Optical Sciences, University of \\ Arizona, Tucson, AZ, USA; ${ }^{\mathrm{d} U S D A}$-ARS Arid Land Research Center, Maricopa, AZ, \\ USA; ${ }^{\circ}$ CSUMB/NASA Ames Research Center, Moffett Field, CA, USA
}

\begin{abstract}
Remotely sensing plant canopy water status remains a long term goal of remote sensing research. Established approaches to estimating canopy water status - the Crop Water Stress Index, the Water Deficit Index, the Equivalent Water Thickness and the many other indices - involve measurements in the thermal or reflective infrared. Here we report plant water status estimates based upon analysis of polarized visible imagery of a cotton canopy measured by Ground Multiangle SpectroPolarimetric Imager (GroundMSPI). Such estimators potentially provide access to the plant hydrological photochemistry that manifests scattering and absorption effects in the visible spectral region.
\end{abstract}

\section{INTRODUCTION}

Remotely sensing the water status of plants and the water content of canopies remain long term goals of remote sensing research [1][2]. Estimates of canopy water status may involve measurements in the thermal infrared portion of the optical spectrum and the Crop Water Stress Index (CWSI) [3] [4] - or its improvement, the Water Deficit Index (WDI) [5]. More commonly, estimates of canopy water status involve measurements in the 900-2000 nm reflective infrared portion of optical spectrum and an index such as the Equivalent Water Thickness (EWT) [6] or its peers [1] [2].

CWSI, the first widely adopted remote sensing plant water stress index, is tied to plant physiology, its principle advantage. It provides indication whether plant stomata are open or closed based upon the principles of evaporative cooling and the foliage radiant temperature relative to the surrounding air temperature. CWSI theory assumes a closed canopy having wall-to-wall vegetation and no soil visible from above the canopy. Moran et al. [5] modified and extended the CWSI theory, proposing the Water Deficit Index in order to account for effects due to the bare soil, often visible between rows of plants in agricultural crop canopies and between the vegetation patches common to dry environments.

Limitations affect CWSI/WDI popularity and applicability. First, it does not work well in regions with high humidity where evaporative cooling effects may be limited. Second, its use requires estimates of the water vapor pressure deficit during the remote sensing over-flight; weather stations that provide such estimates are not always helpfully located next to the canopy. Third, once water-stressed plant canopy stomata close, CWSI/WDI will indicate that the canopy is indeed water-stressed but not how close that canopy is to its permanent wilting point (when plant cells die). Finally, its use requires analysis of calibrated, atmospherically corrected, thermal infrared data, which are not always readily available.

The Equivalent Water Thickness (EWT) established the mathematical linkage between remote sensing observations, the physics of light absorption by water in the 900-20000 nm spectral region, and the canopy water content [6]. Numerous additional indices implicitly based upon this physics have been proposed [1] [2]. Estimates of EWT equate the water in a canopy to a hypothetical horizontal layer of water [6]. Atmospherically corrected, calibrated, reflective infrared measurements are easily obtained, a key EWT advantage compared to CWSI/DWI, but depend upon the physics of water-light interaction, not upon plant physiology - which is the key limitation with EWT. Without a priori information, it is not possible to

Polarization Science and Remote Sensing VIII, edited by Joseph A. Shaw, Frans Snik, Proc. of SPIE Vol. 10407, 104070Z · @ 2017 SPIE · CCC code: 0277-786X/17/\$18 doi: $10.1117 / 12.2274502$ 
determine from estimates of canopy EWT if plants displaying a specific EWT are, for example, satiated or at their permanent wilting point or somewhere in between. Thus, EWT provides estimates of canopy water content rather than canopy water status. However, it should be noted that canopy water status often can be inferred when EWT estimates are interpreted based upon other remote sensing results.

In addition to CWSI/WDI and EWT estimators, plant canopy water status may be estimated using the Photochemical Reflectance Index (PRI) and fluorescence [7] [8], although neither enjoys the popularity nor the robustness of the CWSI/WDI and EWT approaches. A key issue with both is that any observed change in PRI or fluorescence has not one but many potential causes [8]. That is, if the canopy PRI value or the amount of fluorescent light emitted by a canopy changes, the potential cause of that change could be a change in plant canopy water status - or incident light level, nutrient availability, ambient temperature, wind speed and direction or other factor that affects the plant photochemistry. However, the additional information needed to attribute one cause to an observed change is sometimes available; the case of carefully managed, irrigated, agricultural plant canopies provides one example.

Visual indicators closely linked to plant water status have been reported. For example, leaves, when waterstressed, may droop or curl into a tube (canopy architectural changes) and appear gray (a spectral light scattering change) compared to fully hydrated leaves that typically appear turgid and vivid green. However, robust, widely accepted remotely sensible metrics that exploit such visual indicators for estimating canopy water status are not available.

In prior research we reported a linear relationship between the light reflected by the interiors of individual corn leaves measured in vivo and the leaf relative water content (RWC), $\mathrm{R}^{2}=0.77$ [9]. In our recent research [10], we reported a linear relationship between RWC and the ratio

$$
\frac{[\text { light reflected by leaf interior }]}{[\text { light transmitted by leaf }]}
$$

Here we report results of our continuing search for robust estimators of plant canopy water status based upon remotely sensible measurements of the visible and near infrared spectral regions. Such estimators potentially would provide access to the plant hydrological photochemistry that occurs in the 400-800 nm spectrum. We used the GroundMSPI (Ground Multiangle SpectroPolarimetric Imager) [11] system to remotely sense a cotton canopy at the leaf-scale, allowing us to extend our laboratory research to the canopy-scale and monitor both the sunlit leaf RWC and the light scattered by the sunlit leaf interior. We anticipate the knowledge gained at the canopy sunlit leaf-scale will be applicable to analysis of the mixed pixels common to remotely sensed imagery from aircraft and satellite sensors.

\subsection{Overview}

\section{METHODS}

Oblique multispectral polarized imagery was collected of cotton plants (Gossypium spp.) in a test plot, Fig. 1 , within a larger irrigated cotton field during morning and afternoon data collection windows approximately $+/-3$ hours from local solar noon on September 11, 2015, at the United States Department of Agriculture Arid Land Agricultural Research Center, Maricopa, Arizona using GroundMSPI mounted on a tripod in the bed of a pickup truck, Fig. 2. Leaf RWC was estimated from measurements of two sunlit leaves harvested in each row, Fig. 1, during both the morning and afternoon collection windows. Plants in the cotton field were scheduled to be irrigated September 11, but the normal irrigation cycle was delayed for purposes of conducting our experiment; leaf droop indicative of water stress was evident in the test plot during the afternoon of September 11. To obtain a wider range of values, we applied water to three furrows in each of two sections of the test plot, Figs. 1 and 3a, late in the afternoon September 10. Water percolation into the sandy clay loam soil, Fig. 3b, required approximately one hour. Cotton plants were in the boll development stage with the number of open bolls in the canopy beginning a rapid increase. 


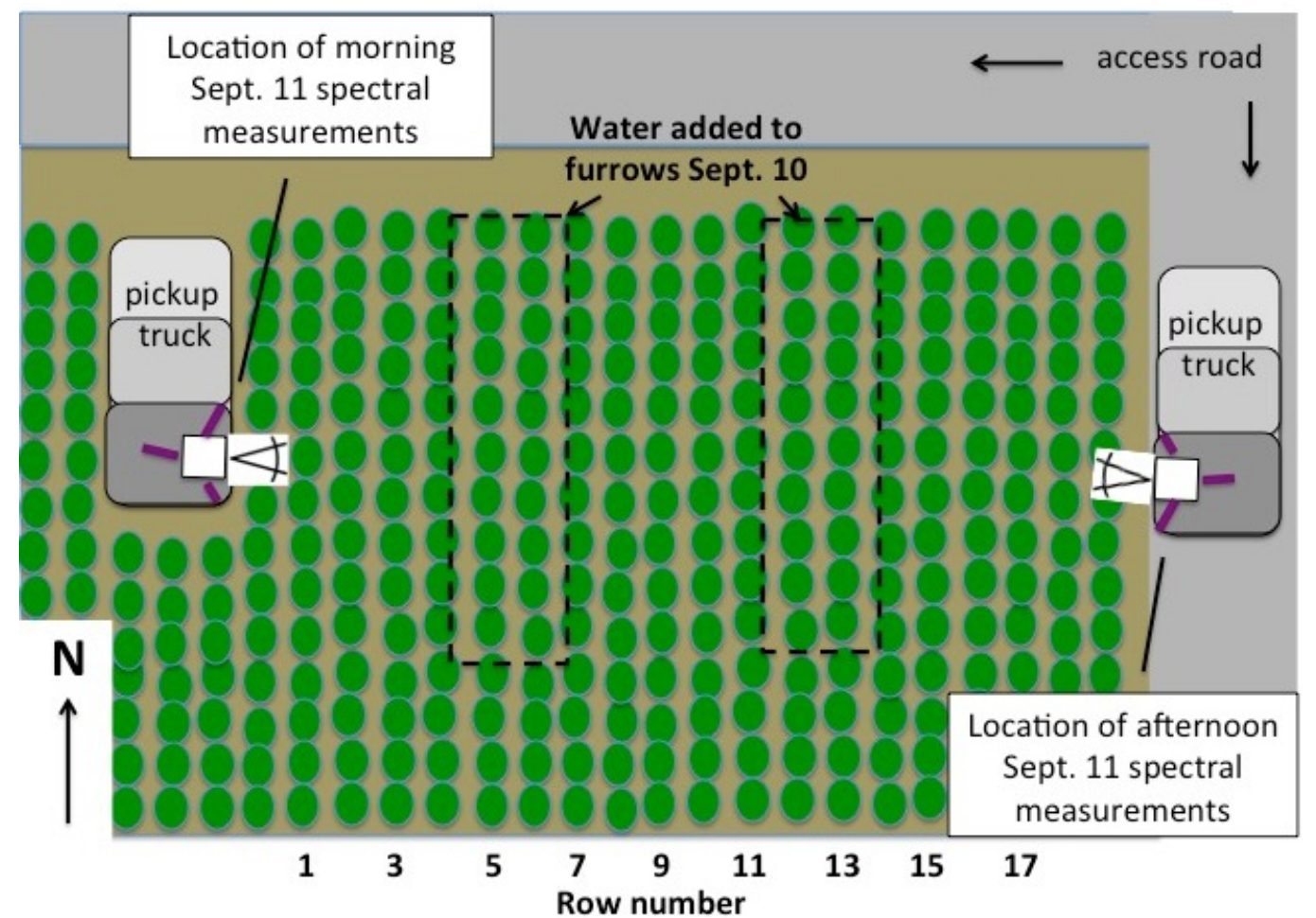

Figure 1. Plan view of the test plot for our cotton experiment investigating leaf relative water content v. leaf and plant optical properties.

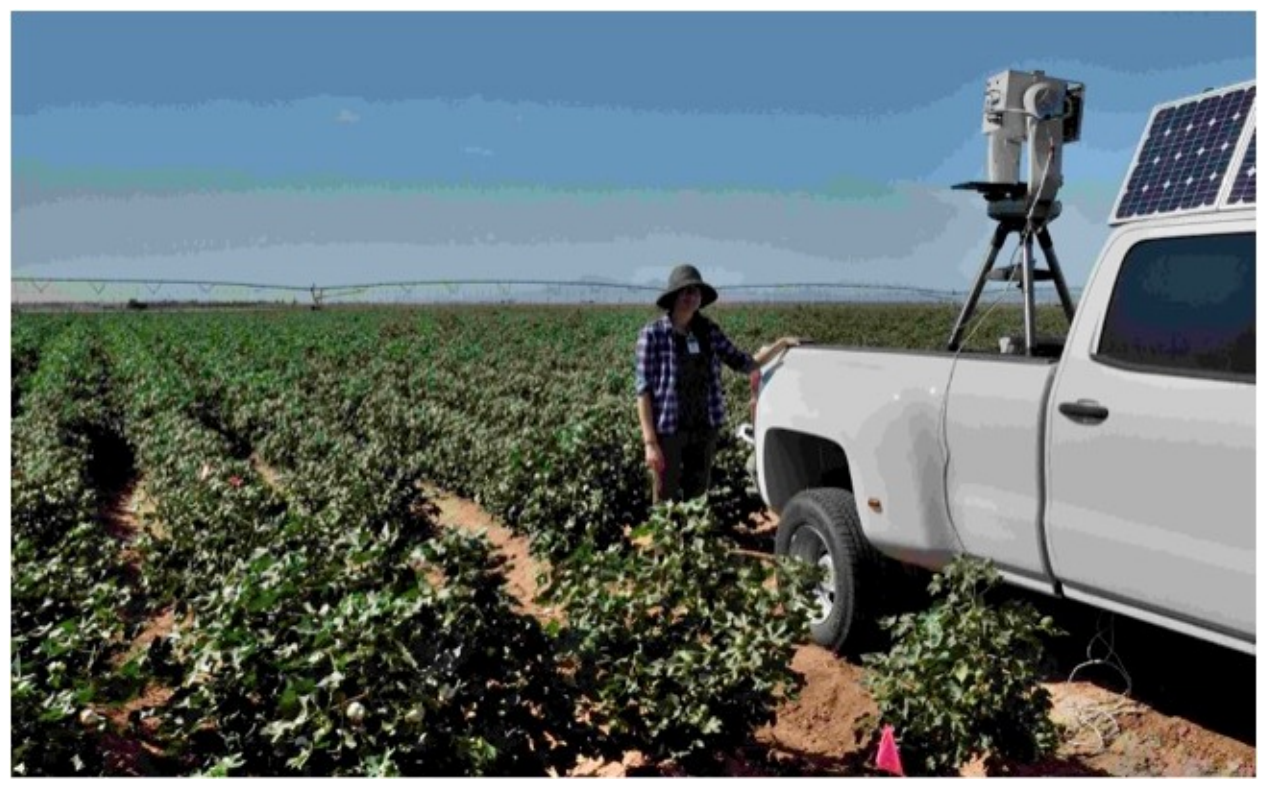

Figure 2. The GroundMSPI instrument, mounted atop a tripod in the bed of a pickup truck, was used to collect oblique multispectral polarized imagery of cotton plants in the test plot during morning (shown here) and afternoon data collections. 


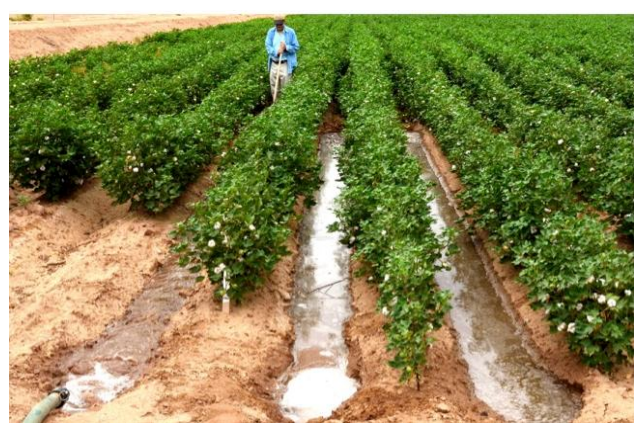

(a)

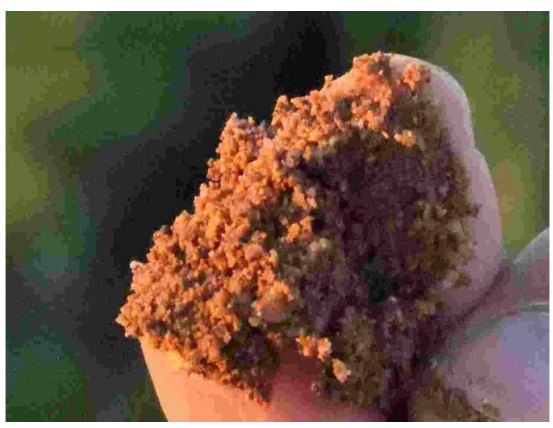

(b)

Figure 3. (a) Three furrows in each of two sections of the test plot were flood-irrigated late in the afternoon of September 10. Following overnight plant hydration, all spectral imagery and RWC data were collected the next day, September 11. (b) Sand particles are apparent in a 'pinch' of the sandy clay loam soil from the test plot.

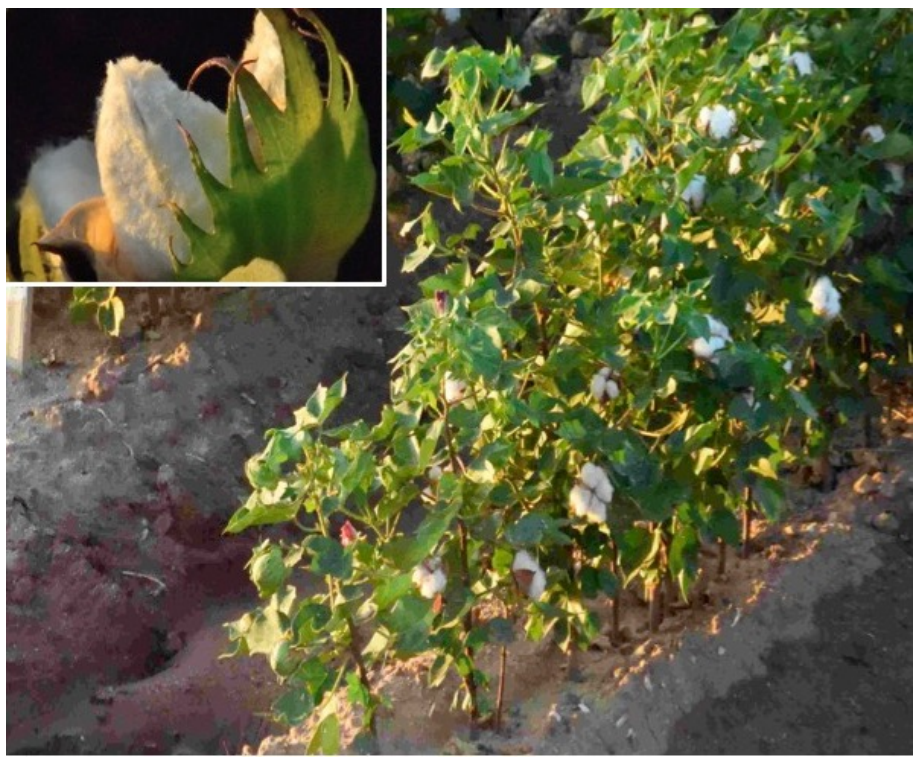

Figure 4. Cotton plants were in the boll development stage with open bolls apparent on many, but not all plants.

\subsection{Polarized multispectral imagery}

\subsubsection{Image preprocessing}

\subsubsection{Sunlit leaf interior reflectance}

We shall estimate the directional hemispherical reflectance factor, $\rho_{i}$, representing the response of the interior of a leaf to sunlight that both enters and then exits the leaf interior through its adaxial or top surface. Use of the well-established directional hemispherical reflectance nomenclature [12] allows comparisons between leaves across the cotton test plot and with results obtained elsewhere. We shall analyze only those shiny bright leaf areas, Fig. 5, that specularly redirect sunlight toward GroundMSPI. As Fig. 5 shows, this represents only a fraction of all the leaf area in the cotton canopy. The light specularly reflected by these leaf areas is both bright and polarized, provided the sensor view direction is not in or near the canopy hot spot direction (i.e. where angle of incidence of sunlight on the leaf area is $0^{\circ}$ ), nor near view directions toward the sun (i.e. where angle of incidence is $90^{\circ}$ ). We are able to identify these bright 
specularly reflecting leaf areas in canopy imagery as pixels having magnitudes greater than a relatively large threshold value.
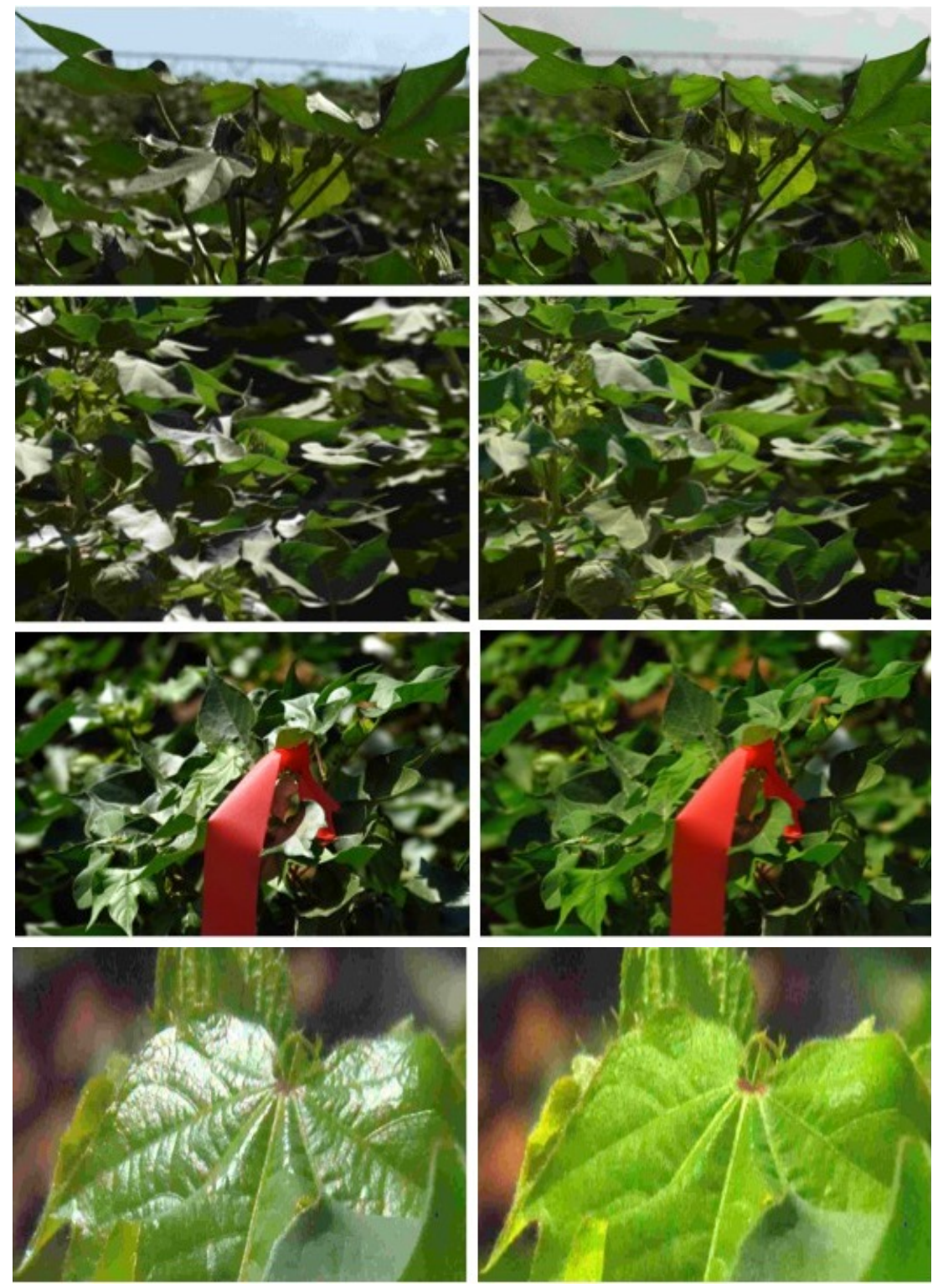

Figure 5. The white surface reflection on cotton leaves was maximized (left column) and minimized (right column) with the aid of a polarization filter rotated in front of the camera lens. The red ribbon allows identification of a RWC leaf harvest location in polarized multispectral imagery of the canopy.

We now develop an analysis procedure for one pure pixel and apply it to other pixels. The selected pure pixel represents a specularly reflecting leaf surface area $\Delta A$ imaged by the GroundMSPI sensor. The mere fact that the leaf area $\Delta A$ specularly redirects sunlight to the sensor establishes the direction of the vector normal to $\Delta A$, and most importantly, the angle of incidence, $\gamma$, of sunlight on it; both may be calculated from two angular directions: the direction from the leaf area $\Delta A$ to the sun and from the leaf area $\Delta A$ to the sensor [9]. 
GroundMSPI imagery provides estimates for each pixel of the three Stokes Vector components - $I, Q$ and $U$ - where $I$ is the intensity; we let $P$ be the polarized intensity, $P=\sqrt{ }\left(Q^{2}+U^{2}\right)$. We divide $I$ for our pure pixel into its specular $S$ and diffuse $D$ components using the procedure described in [13], [14] and a) an assumed value of 1.5 for the index of refraction of the epicuticular wax layer that covers all cotton leaves, b) the angle of incidence (as estimated above) and c) the polarized intensity $P$ for our pure pixel obtained from GroundMSPI imagery. Subsequently, with the newly calculated specular intensity $S$ in hand, we estimate the diffuse intensity $D$ :

$$
D=I-S
$$

Dimensions of $I, P, D$ and $S$ may be either watts $/ \mathrm{sr}$ or watts $/ \mathrm{cm}^{2}$-sr. We shall assume there was negligible light diffusely scattered by the surfaces of the cotton leaves in our test plot; in other words, the leaves appeared shiny and, for purposes of developing the theory here, we ignore leaf surface dust and pubescence that, in fact, appeared negligible, Fig. 5. Therefore, we argue that the diffuse intensity $D$ corresponding to $\Delta A$ represents that portion of the incident sunlight that first enters and then exits the leaf interior through its adaxial surface. Continuing with $D$, we shall now calculate the directional hemispherical reflectance of the leaf interior.

We divide $D$ by the cosine of the angle of incidence, $\gamma$, thereby removing variation in $D$ due to the angle of the solar irradiance on the leaf area $\Delta A$. This in effect normalizes $D$ to normal incidence illumination. We shall assume the cotton leaf interiors in the canopy are Lambertian light scatterers [15]. This allows us to change $D$ to a directional hemispherical quantity by multiplying by $\pi$ sr. Finally, we calculate a reflectance by calibrating $D$ with reference to the hypothetical hemispherical measurement of a perfectly white, perfectly diffuse calibration surface that replaces our leaf area $\Delta A$, and whose surface normal vector points toward the sun. We estimate this hypothetical hemispherical measurement starting from the measurement, $I_{c a l}$, of a second hypothetical calibration surface - this one horizontal and also perfectly white and perfectly diffuse - multiplying by $\pi \mathrm{sr}$ and dividing by cosine of the solar zenith angle, $\theta_{\text {Sun. }}$. Thus, our estimate of the normal incidence, directional hemispherical reflectance of the leaf interior beneath our leaf area $\Delta A$ is found

$$
\rho_{i}=\frac{D\left[\frac{\pi}{\cos \gamma}\right]}{I_{\text {cal }}\left[\frac{\pi}{\cos \theta_{\text {sun }}}\right]}
$$

or

$$
\rho_{i}=\frac{D}{I_{\text {cal }}}\left[\frac{\cos \theta_{\text {sun }}}{\cos \gamma}\right]
$$

This estimate of $\rho_{i}$ is based upon analysis of canopy polarization imagery that resolves individual leaves thereby allowing analysis of those pure pixels that represent leaf area specularly redirecting sunlight to the sensor. Note also that the directional hemispherical reflectance of a leaf measured using an integrating sphere equipped spectrometer includes contributions not only from the leaf interior, but also the leaf surface and thus does not equal $\rho_{i}$, which only represents the leaf interior.

2.2.3 Sunlit leaf transmittance We shall estimate the leaf directional hemispherical transmittance factor, $\tau$, for each row in the canopy, a choice suggested by the fact that we have per row estimates of sunlit leaf RWC (see section 2.3 below). We apply classification algorithms to the polarized multispectral imagery in order to identify the many pixels that represent sunlit transmitting leaf area within the selected canopy row. For these pixels, the intensity $I_{\text {trans }}$ depends upon the angle of incidence of the sunlight on the leaf. From a histogram of $I_{\text {trans }}$ for the row, we select those with the largest magnitudes, making the assumption those represent leaf areas having angles of incidence nearly $0^{\circ}$. Note that here we are implicitly assuming the sunlit leaf areas in the row transmit sunlight similarly. In order to account for variation - noise - in the $I_{\text {trans }}$ values, we quite arbitrarily discard the three largest $I_{\text {trans }}$ values and average the remaining three largest 
$I_{\text {trans }}$ values - in order to obtain an estimate of $I_{\text {trans, } \max }$ that represents the sunlight transmitted by a leaf area illuminated at $0^{\circ}$ angle of incidence. Assuming leaf transmittance is Lambertian [15] allows us to change $I_{\text {trans, } \max }$ to a directional hemispherical quantity by multiplying by $\pi$ sr. Finally, we obtain the leaf directional hemispherical transmittance factor, $\tau$, for each row by calibrating $I_{\text {trans, max }}$ following the procedure used for $\rho_{i}$, obtaining

or

$$
\tau=\frac{\pi I_{\text {trans, } \max }}{I_{\text {cal }}\left[\frac{\pi}{\cos \theta_{\text {sun }}}\right]}
$$

$$
\tau=\frac{\cos \theta_{\text {sun }} I_{\text {trans,max }}}{I_{\text {cal }}}
$$

as the directional hemispherical transmittance factor of sunlit leaves. And we point out, this analysis approach will only succeed if the imaging sensor field of view includes sunlit transmitting leaf area with a $0^{\circ}$ angle of incidence. The directional hemispherical transmittance of a leaf measured using an integrating sphere equipped spectrometer equals $\tau$ obtained here.

\subsubsection{Conservation of energy}

The light hemispherical scattering and absorbing properties of a sunlit leaf may be summarized

$$
1.0=\rho_{s}+\rho_{i}+\tau+\alpha
$$

where the hemispherical reflectance of the leaf surface, $\rho_{s}$ (defined here to include only light that never enters the leaf interior), the hemispherical reflectance of the leaf interior, $\rho_{i}$, the leaf hemispherical transmittance and the leaf absorption all sum to 1.0.

\subsubsection{Remote sensing estimates of canopy RWC}

In this section 2.2 we have proposed methodology to obtain estimates of the light reflected and transmitted by leaf interiors all based upon analysis of polarized multispectral imagery of a cotton canopy measured by GroundMSPI. Our prior research at the leaf-scale, [9], [10], has reported linear relationships between RWC and both the reflectance of the leaf interior, and the ratio of the leaf interior reflectance and leaf transmittance. We intend to explore the cotton canopy data looking for similar relationships between RWC and the reflectance and transmittance variables developed in this section 2.2.

\subsection{Relative water content}

We estimated the leaf Relative Water Content for each row 1-17, Fig. 1, during morning and again during afternoon data collection periods. We harvested two sunlit leaves samples in each row, immediately placed each sample with cut end down in a tube in an ice chest partially filled with ice and transported the chest to the lab. We weighed each tube + leaf sample, then rehydrated each sample overnight, immersing the leaf cut end in water in its tube. The next morning, we removed each now fully hydrated leaf sample from its tube, blotted the sample dry, weighed it, then placed it in an envelope in a $65^{\circ} \mathrm{C}$ drying oven for two days, cooled and weighed the leaf samples and estimated RWC for a specific leaf weight as

$$
R W C=\frac{[\text { leaf wt. }]-[\text { leaf dry wt. }]}{[\text { leaf fully hydrated wt. }]-[\text { leaf dry wt. }]}
$$

The RWC of each row was calculated as the average of the two leaf sample RWCs for that row. 


\subsection{Relative water content}

\section{RESULTS AND DISCUSSION}

The results, Fig. 6 , show that RWC values in most rows were between 0.8 and 0.9 during the morning data collection window and less than 0.85 during the afternoon window. While we did not measure the RWC corresponding to zero turgor within the cells of the cotton leaves, experience suggests it perhaps is around RWC $\sim 0.85$. The point of zero turgor, when hydrostatic pressure inside leaf cells is zero - as well as the RWC corresponding to zero turgor - provide important insight into leaf water status. As leaf RWC decreases past the point of zero turgor, leaf stomata - the tiny gas exchange openings on the leaf surface begin closing. While this helps the leaf cells retain water, the resulting cutoff of $\mathrm{CO} 2$ causes cell photosynthesis to stop. If zero turgor in the cells of the cotton leaves did indeed occur around an RWC 085 , then during morning data collection only the watered plants were photosynthesizing, while during afternoon data collection photosynthetic activity in all plants was limited.

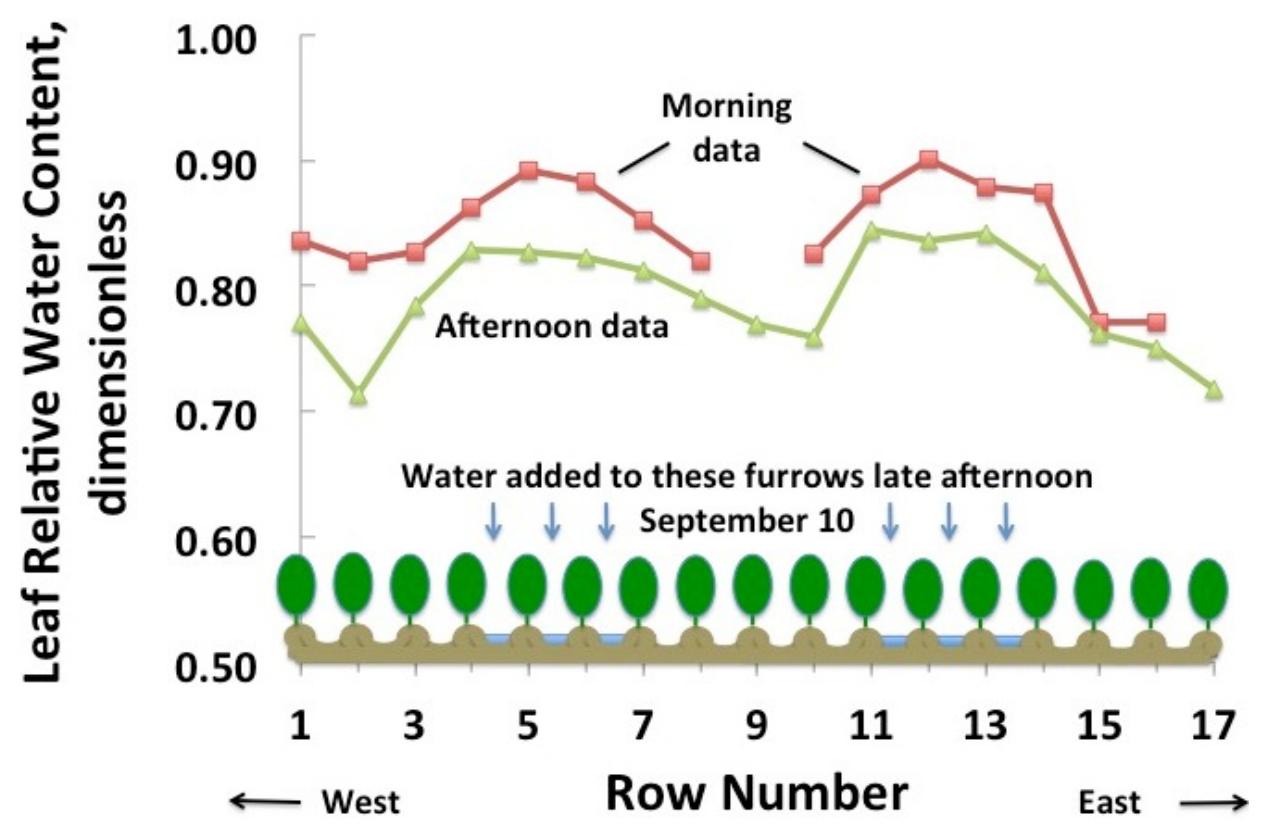

Figure 6. Leaf RWC decreased from morning to afternoon and varied by row number, Fig. 1.

The results, Fig. 7, reveal the irrigated plants in rows 11-14 appear a different shade of green than nonirrigated plants. The number of bright, polarized sun glints from leaves appears reduced in the irrigated rows, suggesting that the leaf angle distributions for the irrigated and non-irrigated plants are different. We believe these canopy architectural differences are due in part to the effects of leaf droop in the non-irrigated portion of the test plot. The analysis methods, described in section 2.2, for estimating the directional hemispherical reflectance of the leaf interior are applicable to bright, highly polarized, specularly reflecting leaf areas; the analysis methods are not applicable if no such bright leaf areas appear in canopy imagery. 

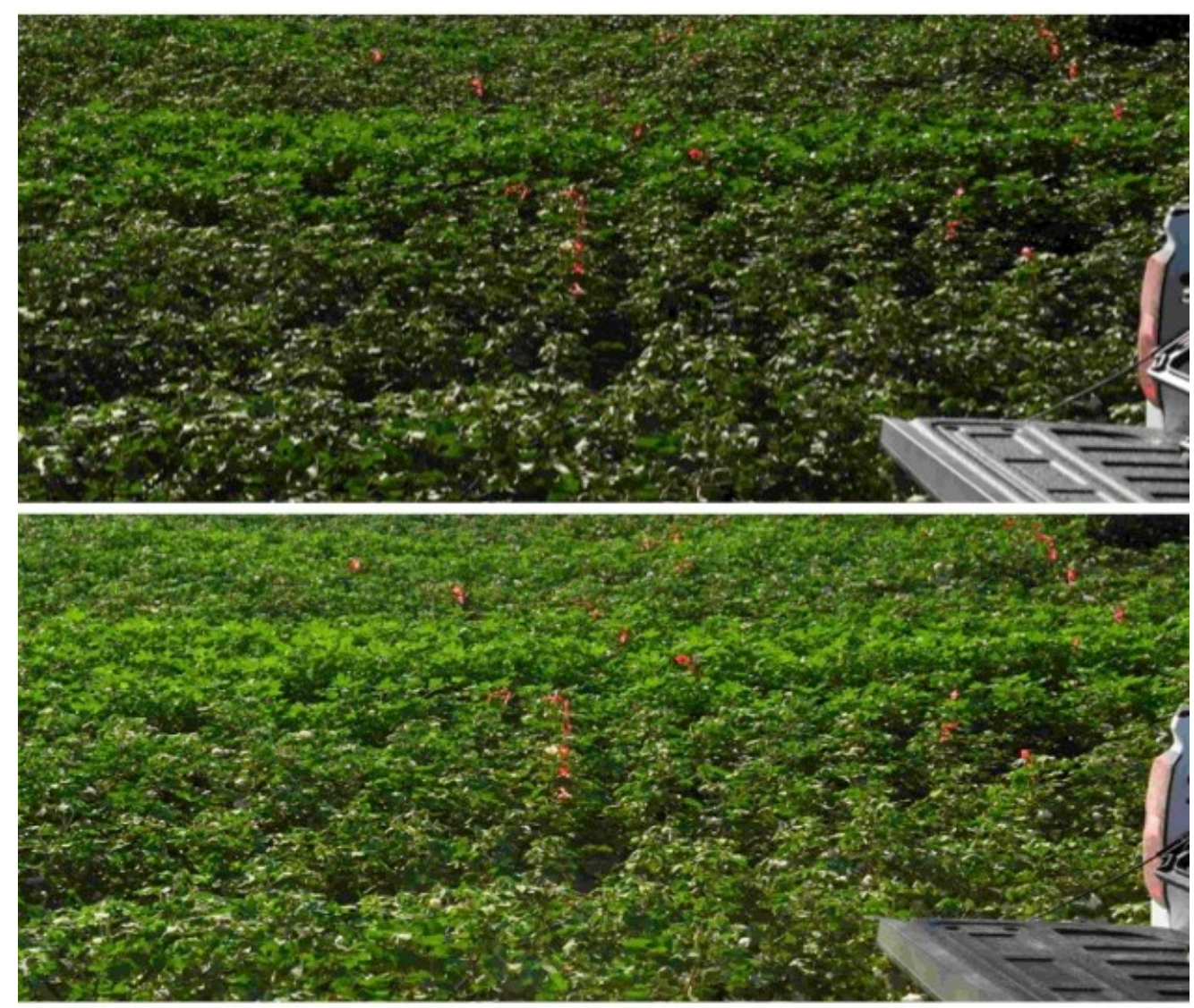

Figure 7. Photos with polarization filter rotated for maximum (top) and minimum (bottom) leaf surface reflection. View is west from near the afternoon spectral imagery collection location. Reflection from tailgate of pickup truck appears partially polarized. Plants in rows 11-14 irrigated September 10 appear as a lighter green horizontal band approximately $1 / 4$ of the distance from top to bottom of each photo. Red ribbons allow identification of RWC leaf harvest locations in polarized multispectral imagery of the canopy.

\section{CONCLUSIONS}

We have presented a methodology, described in section 2.2, for analysis of polarized multispectral plant canopy imagery in order to estimate sunlit leaf relative water content (RWC) for a canopy. The methodology represents the application/modification of our published leaf-scale methodology to the canopy-scale. The approach depends in part upon canopy leaves specularly reflecting sunlight to an imaging sensor.

Here we propose only a methodology; we do not report here results of the application of this methodology to estimating sunlit leaf RWC for a cotton canopy. We anticipate being able to present orally at the SPIE Polarization and Remote Sensing VIII Conference to be held in San Diego, August 89, 2017, results of the application our proposed canopy-scale methodology to analysis of GroundMSPI imagery of a cotton canopy. 


\section{REFERENCES}

[1] Ustin, S.L., D. Riaño, D. and Hunt, E.R., "Estimating canopy water content from spectroscopy," Israel Journal of Plant Sciences, 60, 9-23 (2012).

[2] Roberto, C., Busetto L., Meroni M., Rossini M., and Panigada C., "Optical Remote Sensing of Vegetation Water Content," in Hyperspectral Remote Sensing of Vegetation, P.S. Thenkabail, J.G. Lyon, and A. Huete, Eds., CRC Press, Boca Raton, London, New York, ch. 10, pp 227-244, (2011). ISBN: 978-14398-4537-0

[3] Idso, S.B., Jackson, R.D., Pinter Jr., P.J., Reginato R.J. and Hatfield, J.L., "Normalizing the stressdegree-day parameter for environmental variability," Agricultural Meteorology, 24, 45-55 (1981).

[4] Jackson, R.D., Idso, S.B., Reginato R.J. and Pinter, Jr., P.J., “ Canopy temperature as a crop water stress indicator," Water Resources Research. 17, 1133-1138 (1981).

[5] Moran, M.S., Clarke, T.R., Inoue Y. and Vidal, A., "Estimating crop water deficit using the relation between surface-air temperature and spectral vegetation index," Remote Sensing of Environment. 49, 246-263 (1994).

[6] Gao B.C. and Goetz, A.F.H., "Retrieval of equivalent water thickness and information related to biochemical components of vegetation canopies from AVIRIS data," Remote Sensing of Environment., 52, 155-162 (1995).

[7] Baker, N.R., "Chlorophyll Fluorescence: A Probe of Photosynthesis In Vivo." Annual Review of Plant Biology. 59, 89-113 (2008).

[8] Sarlikioti, V., Driever S.M. and Marcelis, L.F.M, "Photochemical reflectance index as a means of monitoring early water stress.” Annals of Applied Biology. 157, 81-89 (2010).

[9] Vanderbilt, V.C., Grant L. and Daughtry, C.S.T., "Polarization of light scattered by vegetation." Proceedings of the IEEE, 73, 1012-102 (1985).

[10] Vanderbilt, V., Daughtry, C. and Dahlgren, R., "Leaf relative water content estimated from leaf reflectance and transmittance." IEEE Geoscience and Remote Sensing Symposium (IGARSS), 10-15 July 2016. ISSN: 2153-7003, INSPEC Accession Number: 16445241, DOI: $\underline{10.1109 / \text { IGARSS.2016.7730870 }}$

[11] Diner, David J., et al., "First results from a dual photoelastic-modulator-based polarimetric camera." Applied Optics 49, 2929-2946 (2010)

[12] Nicodemus, F.E., Richmond, J.C., Hsia, J.J., Ginsberg, I.W., Limperis, T., "Geometrical considerations and nomenclature for reflectance.” National Bureau of Standards, NBS MN-160. (1977).

[13] Vanderbilt, V.C. and Grant, L., "Plant canopy specular reflectance model." IEEE Transactions on Geoscience and Remote Sensing, 5, 722-730 (1985).

[14] Vanderbilt, V.C. and De Venecia, K.J., "Specular, diffuse, and polarized imagery of an oat canopy." IEEE Transactions on Geoscience and Remote Sensing, 26, 451-462 (1988).

[15] Breece, H.T. and Holmes, R.A., "Bidirectional scattering characteristics of healthy green soybean and corn leaves in vivo.” Applied Optics, 10, 119-127 (1971). 